\title{
MONITOR Ionospheric Network: two case studies on scintillation and electron content variability
}

\author{
Yannick Béniguel $^{1}$, Iurii Cherniak ${ }^{2}$, Alberto Garcia-Rigo ${ }^{3}$, Pierrick Hamel ${ }^{1}$, Manuel Hernández-Pajares ${ }^{3}$, \\ Roland Kameni $^{4}$, Anton Kashcheyev ${ }^{5}$, Andrzej Krankowski ${ }^{2}$, Michel Monnerat ${ }^{8}$, Bruno Nava ${ }^{5}$, Herbert Ngaya ${ }^{4}$, \\ Raül Orus-Perez ${ }^{6}$, Hughes Secrétan ${ }^{7}$, Damien Sérant ${ }^{8}$, Stefan Schlüter $^{9}$, and Volker Wilken ${ }^{10}$ \\ ${ }^{1}$ Informatique, Electromagnétisme, Electronique, Analyse numérique (IEEA), Courbevoie, 92400, France \\ ${ }^{2}$ University of Warmia and Mazury, Olsztyn, Poland \\ ${ }^{3}$ UPC-IonSat, Universitat Politècnica de Catalunya, Barcelona, 08034, Spain \\ ${ }^{4}$ Agence pour la sécurité de la navigation aérienne en Afrique et à Madagascar (ASECNA), Dakar, Senegal \\ ${ }^{5}$ International Center for Theoretical Physics (ICTP), Trieste, 34014, Italy \\ ${ }^{6}$ European Space Agency/European Space Research and Technology Center (ESA/ESTEC), Noordwijk, \\ $2201 \mathrm{AZ}$, the Netherlands \\ ${ }^{7}$ Centre National d'Etudes Spatiales (CNES), Toulouse, 31401, France \\ ${ }^{8}$ Thales Alenia Space France, Toulouse, 31037, France \\ ${ }^{9}$ European Space Agency/EGNOS Project Office (ESA/EPO), Toulouse, 31401, France \\ ${ }^{10}$ Deutsches Zentrum für Luft- und Raumfahrt (DLR), Neustrelitz, 17235, Germany
}

Correspondence to: Yannick Béniguel (beniguel@ieea.fr)

Received: 31 July 2016 - Revised: 23 January 2017 - Accepted: 1 February 2017 - Published: 13 March 2017

\begin{abstract}
The ESA MONITOR network is composed of high-frequency-sampling global navigation satellite systems (GNSS) receivers deployed mainly at low and high latitudes to study ionosphere variability and jointly with global GNSS data and ionospheric processing software in support of the GNSS and its satellite-based augmentation systems (SBAS) like the European EGNOS. In a recent phase of the project, the network was merged with the CNES/ASECNA network and new receivers were added to complement the latter in the western African sector. This paper summarizes MONITOR, presenting two case studies on scintillations (using almost 2 years of data measurements). The first case occurred during the major St. Patrick's Day geomagnetic storm in 2015. The second case study was performed in the last phase of the project, which was supported by ESA EGNOS Project Office, when we paid special attention to extreme events that might degrade the system performance of the European EGNOS.
\end{abstract}

Keywords. Ionosphere (ionospheric irregularities)

\section{Introduction}

MONITOR (MONitoring of Ionosphere by innovative Techniques coordinated Observations and Resources) (Béniguel et al., 2015) is a project conducted by the European Space Agency's GNSS Evolution Programme. It is dedicated to the collection, processing, and archiving of ionospheric data and products during active periods of solar activity; the development of improved scintillation monitoring instrumentation; and the establishment of a scintillation monitoring network in order to build the appropriate infrastructure for analysing the impact of the ionosphere on European GNSS (EGNOS and Galileo) system performance. The receivers deployed for the project have been installed both in low and high latitudes, which are the two main regions where the ionosphere might be the cause of EGNOS decreases in performance. The lowlatitude sector is also of interest for evaluating the constraints for a possible extension of EGNOS in Africa in the future.

The ionosphere MONITOR stations (IMONIS) are equipped with a $50 \mathrm{~Hz}$ scintillation receiver (Septentrio PolaRxS or NovAtel 4004B) and bit grabbers in order to record IF data beyond the tracking capability of GNSS receivers for 
later analysis in a laboratory environment. At low latitudes, the stations in western Africa were deployed to complement the five stations of the CNES/ASECNA SAGAIE network (Stations ASECNA GNSS pour l'Analyse de l'Ionosphère Equatoriale) (Secrétan et al., 2014). This was done in mid2015. Both the MONITOR and SAGAIE stations in Africa are shown in Fig. 1. All low-latitude stations have Septentrio PolaRxS receivers. At high latitudes there are three stations, in Kevo and Sodankylä in Finland and in Kiruna in Sweden. The receivers used are the PolaRxS in Kevo and Kiruna and the NovAtel 4004B in Sodankylä. The PolaRxS receiver is a modern receiver. It is capable of recording data from the three constellations GPS, GLONASS and Galileo on two frequencies, while the NovAtel receiver corresponds to an old version of this receiver with a reduced capability: only the L1 frequency for the scintillation indices and the GPS constellation.

The results presented in this paper address two issues: the ionospheric scintillation variability and an extreme ionospheric event corresponding to a storm. The scintillation characteristics and their dependency on the geophysical parameters were studied using the data recorded in 2015 and 2016. This represents 1.5 years of high-rate GNSS data for six receivers and almost 2 years for five receivers. The extreme case studied was the St. Patrick's Day magnetic storm of March 2015, which is the most important storm to occur so far during this 24 th solar cycle.

\section{The architecture of the project}

The MONITOR + SAGAIE network was extended in 2015, with five new receivers deployed in the same areas as the first phase of the project at low and high latitudes for an improved accuracy in the scintillation analysis, in particular the latitudinal dependency. At low latitudes, the network extends today from Senegal to Chad, plus one receiver located in Ethiopia. At high latitudes, the network has been extended to the western part of the northern Scandinavian sector.

The MONITOR project also includes a centralized facility that is in charge of collecting and archiving data and products, generating certain additional products and reports, and providing an interface for data provision with partners and third parties. In addition, this facility collects products from processors hosted at external institutions, which are routinely distributed on a periodic basis.

The data collected from the MONITOR and SAGAIE stations are $1 \mathrm{~min}$ ionospheric scintillation indices, receiver independent exchange format (RINEX) files at $1 \mathrm{~Hz}, 50 \mathrm{~Hz}$ raw data and bit grabber IF data. The products are categorized by various types, including

- space weather: solar and geomagnetic indices obtained from third parties.
- station-based: recomputed 1 min ionospheric scintillation indices and losses of lock and cycle slips.

- daily scintillation plots and maps.

- electron content: global electron content, slant total electron content (STEC) values of a subset of International GNSS Service (IGS) receivers, differential STECs (dSTEC truths), vertical TEC (VTEC) global maps, EGNOS VTEC European maps and EGNOS accuracy assessments against independent data.

- perturbations: along-arc TEC rate (AATR) parameter (Sanz et al., 2014) for EGNOS and WAAS reference stations and for the SAGAIE network, rate of TEC, solar flares (Hernández-Pajares et al., 2012a) and medium-scale travelling ionospheric disturbances (MSTIDs, Hernández-Pajares et al., 2012b). The AATR index characterizes the TEC variability. It is an indicator of the hourly RMS of the STEC rate with an appropriate weight function.

- reporting: automatic and manual reports.

As an example, the UPC MONITOR VTEC global maps (see Hernández-Pajares et al., 1999; Orús-Pérez et al., 2005) are provided with high performance (following for instance $\mathrm{Xi}$ ang et al., 2015; Orús-Pérez, 2016; Hernández-Pajares et al., 2016) at a temporal rate of $15 \mathrm{~min}$ (for comparison, IGS provides one or two hourly VTEC maps) and $5^{\circ}$ of longitude and $2.5^{\circ}$ latitude spatial range (see more details in Sect. 5.3).

As an additional activity of the project, a methodology to create ionospheric reference scenarios has been developed. It is based on the NeQuick 2 (Nava et al., 2008) model adaptation of vertical TEC maps to obtain effective ionization parameter (Az) grids (Nava et al., 2011). Using these Az grids as an input for NeQuick allows the model to provide a three-dimensional representation of the electron density of the ionosphere all over the world and to compute STEC values for any ground-to-satellite link.

Using the abovementioned methodology together with the global VTEC maps introduced above with a 15 min time interval produced by the Polytechnical University of Catalonia (UPC), three scenarios were generated:

1. a scenario of 1 month duration, corresponding to a high solar activity period (May 2002);

2. a scenario of 1 month duration, corresponding to a midto-low solar activity period (December 2006);

3. a scenario of 1 week duration, corresponding to a geomagnetically disturbed period (March 2015).

In order to evaluate the proposed ingestion-assimilation method, two different key indicators were considered: the differential slant GPS TEC (dSTEC; as defined for instance by Feltens et al., 2011) and slant GPS TEC calibrated with 


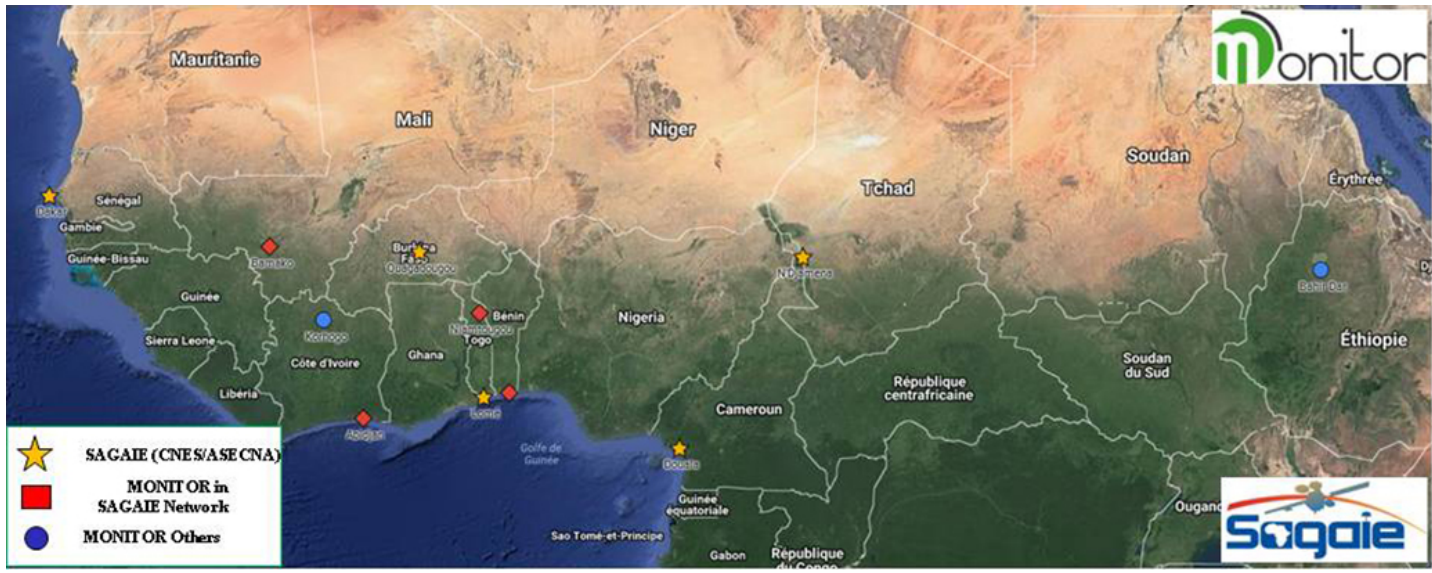

Figure 1. The MONITOR + SAGAIE network (low latitudes).

the UQRG GIMs (STEC). To perform this test, specific MONITOR products (externally provided or derived from internal processors) were used. In particular, VTEC maps with 15 min time intervals produced by UPC were considered for the ingestion, and therefore an Az grid for each VTEC map was calculated. Experimental ground-based dSTECSTEC data at $5 \mathrm{~min}$ time intervals were used as independent "ground truth" measurements for comparison with the corresponding model-retrieved values. For this purpose, the corresponding daily dSTEC and STEC (MUIT/MUST) files produced by UPC were used, and the information about groundreceiver-to-satellite links, including the corresponding experimental dSTEC/STEC, was extracted.

It is understood that the model-retrieved STEC values for the necessary links were calculated with the $\mathrm{NeQuick}$ driven by the relevant Az maps. Finally all the dSTEC-STEC errors were calculated and the corresponding dSTEC-STEC error statistics were computed. A $15^{\circ}$ elevation mask angle was applied to the data for this validation. The relevant dSTEC and STEC error statistics indicate the capability of the proposed data ingestion technique to capture both temporal and spatial gradients of the ionospheric electron density under the helio-geophysical conditions for the periods analysed.

It has to be underlined that experimental data for the validation have not been used for the generation of the VTEC maps used as input for the data ingestion procedure.

\section{Ionospheric scintillations at low and high latitudes}

\subsection{Low-latitude scintillations}

\subsubsection{Latitudinal and seasonal dependencies}

The scintillation data recorded during the MONITOR measurement campaign (hereafter referred as the database) was used to estimate the scintillation area extent and its climatological dependency. The probability of occurrence of scintil-

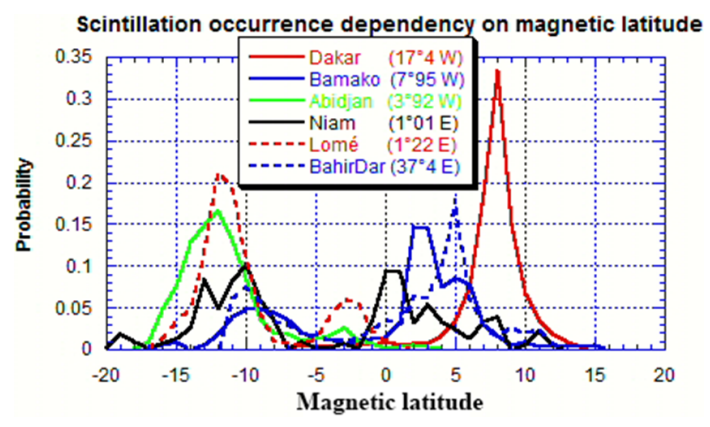

Figure 2. Scintillation probability of occurrence at low latitudes in Africa during the years 2015 and 2016.

lation depending on the magnetic latitude is plotted in Fig. 2. All links exhibiting a value of the amplitude scintillation index (corresponding to the standard deviation of the normalized intensity and noted intensity scintillation index (S4)) greater than 0.2 during the year 2015 were counted to calculate this probability.

The probability curve peaks at $\pm 10^{\circ}$ of the magnetic equator, corresponding to the location of the crests of the equatorial ionosphere. Events between the crests' locations also occur but with a reduced probability. Figure 3 shows the seasonal dependency of scintillations. The probability of occurrence was calculated at each location as the ratio of events cumulated over 1 particular month divided by the total number of events cumulated over the year at the same location. The Dakar and Lomé receivers were operating in 2014. The other stations were deployed in 2015. The first month addressed corresponds to the first month in which the receiver began operating. The conclusion is mixed, as some stations in the Northern Hemisphere (in magnetic coordinates, Dakar in particular) exhibit no scintillation in summer between the equinoxes, while this is not the case in the Southern Hemisphere. The activity is quite similar in summer and winter in 


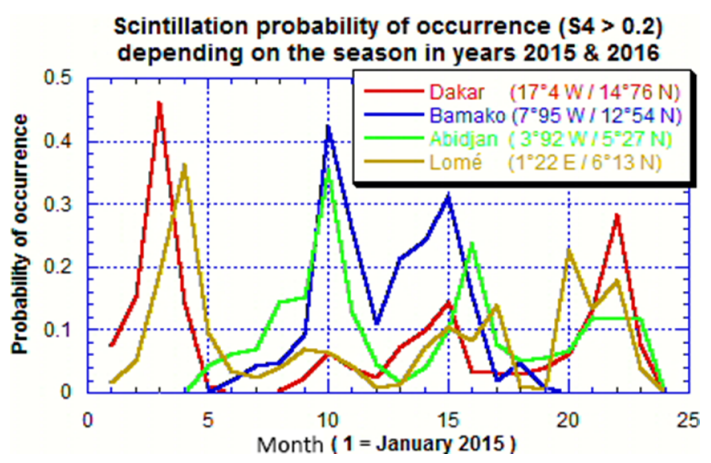

Figure 3. Scintillation probability of occurrence at low latitudes depending on the season.

the Southern Hemisphere. In Lomé this was not the case in 2015, but it was the case in 2016.

Figure 4 is a cumulative map of the scintillations recorded from January to November 2016. The S4 values calculated at receiver level by the manufacturer software and using the $50 \mathrm{~Hz}$ raw data files over $1 \mathrm{~min}$ samples were used to plot this map. The location of the points is the location of the ionospheric pierce points (IPPs), defined as the points where each link crosses the altitude of $350 \mathrm{~km}$. The values of the points in each one of the pixels correspond to the average of the highest $10 \%$ of values of the points attached to this pixel.

This map shall be considered together with the corresponding S4 probabilities shown in Fig. 2. The latitudinal extent appears to be greater in the Southern Hemisphere. Holes in the map both in the northern and southern lines are due to lack of data in the corresponding regions. It is expected in the future to deploy one additional receiver in southern Algeria (Tamanrasset) to complete the northern line of receivers. The code carrier divergence criterion, as proposed by A. J. Van Dierendonck (NovAtel GSV4004B user manual), was used to reject the links affected by multipath. No mask elevation angle was added to this criterion.

\subsubsection{Geophysical dependencies}

Carrano et al. (2016) showed that the phase scintillation index $\sigma_{\Phi}$ is found to be dependent on two parameters: the effective scan velocity of the medium noted $v_{\text {eff }}$ and the cutoff frequency $\tau_{\mathrm{c}}$ of the receiver phase loop. The relationship is as follows:

$\sigma_{\Phi}^{2}=G C_{P} F_{\Phi}(p)\left(v_{\mathrm{eff}} \tau_{\mathrm{c}}\right)^{(p-1)}$.

The effective scan velocity is the velocity of a link crossing the fluctuating medium. It has two components: the medium drift velocity linked to the terrestrial magnetic field and the satellite velocity. Its calculation is detailed at the end of this section. In what follows, the fluctuating medium is assimilated to a single phase screen located at an altitude of $350 \mathrm{~km}$. The point on a link located at this altitude is the IPP and the

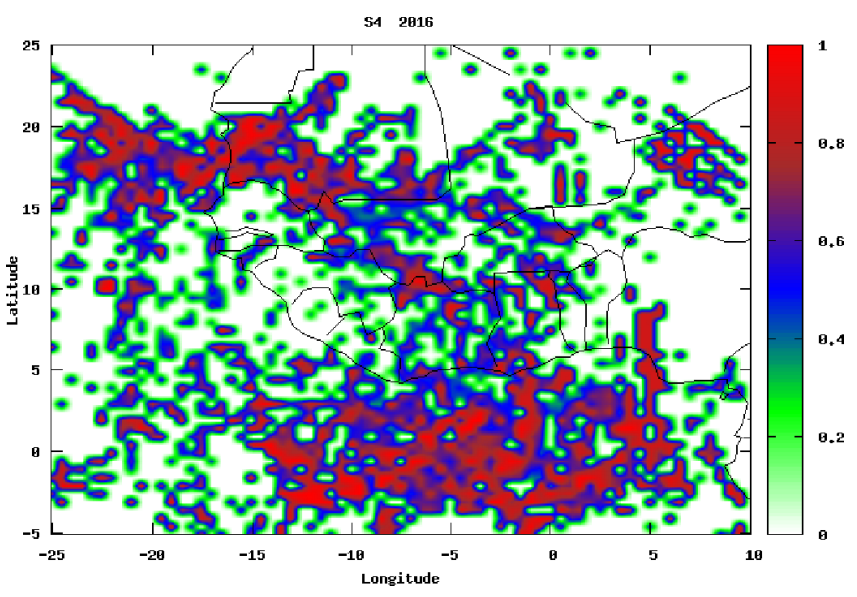

Figure 4. Low-latitude cumulative scintillation map for the first semester of 2016.

IPP velocity is the second component of the effective scan velocity.

The calculation presented hereafter was performed at each IPP in the database in the terrestrial magnetic field reference system attached to that point. $G$ is a geometrical factor (Rino, 1979) given by the relationship

$$
G=\frac{a b}{\sqrt{A C-B^{2} / 4} \cos \vartheta} .
$$

$a$ and $b$ are average values of the lengths of the inhomogeneity axis assumed to be elliptical.

The coefficients $A, B$ and $C$ are geometrical factors included to take the line of sight (LOS) misalignment with the earth magnetic field into account. They involve the earth magnetic declination and inclination angles and the LOS elevation angle $\vartheta$ at each IPP.

$p$ is the slope of the phase spectral density, which is linear in most cases, as a first approximation in a log-log scale representation. It is provided as an output of some commercial receivers, in particular the PolaRxS, using this approximation and processing $1 \mathrm{~min}$ samples of the raw data files.

$C_{P}$ characterizes the strength of the scintillation event. In particular, it includes the thickness of the fluctuating medium and the average size of the inhomogeneities.

The theoretical phase index value is obtained after calculation of the autocorrelation function, which is Fourier transformed from the spectral density. The $F_{\Phi}(p)$ function results from this transformation. It involves a combination of gamma functions of the slope value.

Under the weak fluctuation hypothesis, using the Born approximation, a comparable derivation can be performed to attain the intensity scintillation index, noted as S4. The obtained relationship is

$S_{4}^{2}=C_{p} F_{I}(p) I_{3} \rho_{\mathrm{F}}^{(p-1)}$. 


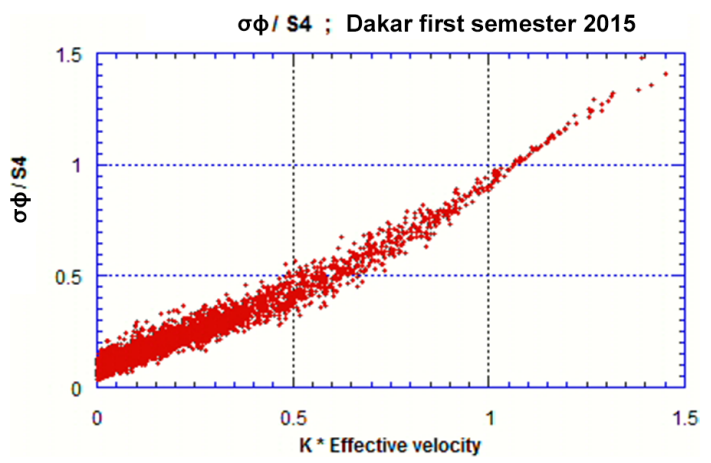

Figure 5. Ratio of intensity to phase scintillation indices in Dakar in 2015 .

The $C_{P}$ factor characterizing the strength of the scintillation also appears in this calculation and will be consequently removed when taking the ratio of the two indices. Same as $F_{\Phi}(p), F_{I}(p)$ is a combination of gamma functions of the slope. $I_{3}$ is an integral term depending on geometrical factors linked to the misalignment of the line of sight with the earth magnetic field. It is evaluated separately.

$\rho_{\mathrm{F}}$ is the Fresnel radius of observations given by the relation $\rho_{\mathrm{F}}=\sqrt{\left(Z_{R} \sec \vartheta\right) /(2 k)}$.

$Z_{R}$ is the IPP altitude set to $350 \mathrm{~km}$ in our calculation and $k$ is the wave number of the operating frequency.

The ratio of the two indices gives the relation

$$
\frac{\sigma_{\Phi}^{2}}{S_{4}^{2}}=\frac{G F_{\Phi}(p)}{I_{3} F_{I}(p)}\left(\frac{\nu_{\mathrm{eff}} \tau_{c}}{\rho_{\mathrm{F}}}\right)^{(p-1)}=H\left(\frac{\nu_{\mathrm{eff}} \tau_{c}}{\rho_{\mathrm{F}}}\right)^{(p-1)} .
$$

The $H$ function has an analytical solution, only related to the slope value $p$ (Carrano et al., 2016).

The relation between the indices above shows the dependencies. This calculation was performed for all links during the first semester of 2015 in Dakar (geographic latitude $14^{\circ} 41^{\prime} \mathrm{N}$ ), irrespective of the S4 value, assimilating the fluctuating medium to a single thin phase screen located at the IPP altitude. The receiver cutoff frequency is $0.1 \mathrm{~Hz}$. Using these values in the equation above leads to a simple relationship depending only on the effective scan velocity. Figure 5 shows the plot obtained for the L1 frequency. The corresponding points are aligned well, showing that the dependencies are identified well. All dependencies except for the effective velocity $v_{\text {eff }}$ are summed up in the constant factor $\mathrm{K}$ on the $x$ axis.

The effective velocity is obtained by reversing the previous equation.

As previously indicated, the phase spectrum exhibits a linear slope in a $\log -\log$ axis representation depending on the operating frequency wave number. In the case of an anisotropic medium, the components of the wave vector shall be included in the calculation. This can be reverted to the autocorrelation function, making it possible to derive a relation between the effective velocity and its components $v_{s x}$ and

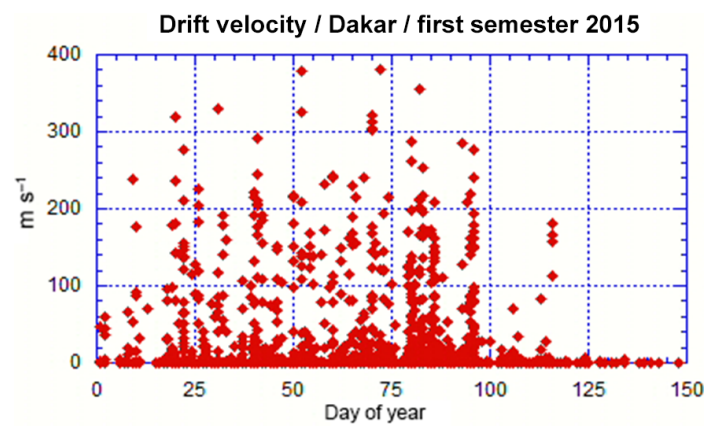

Figure 6. Medium drift velocity in Dakar in 2015.

$v_{s y}$. This calculation was performed by Rino $(1979,2011)$. The obtained relationship is

$v_{\mathrm{eff}}=\left[\frac{C v_{s x}^{2}-B v_{s x} v_{s y}+A v_{s y}^{2}}{A C-B^{2} / 4}\right]^{1 / 2}$,

with $v_{s x}=v_{\mathrm{IPP}, x}-\tan \vartheta \cos \phi v_{\mathrm{IPP}, z}$ and $v_{s y}=v_{\mathrm{IPP}, y}-V_{\mathrm{D}}-$ $\tan \vartheta \sin \phi v_{\mathrm{IPP}, z}$.

$v_{\text {IPP }}$ is the IPP velocity corresponding to the satellite movement on its orbit.

$V_{\mathrm{D}}$ is the medium drift velocity. It is calculated by solving the equations above.

Figure 6 shows the medium drift velocity calculated in Dakar in 2015. There is a direct correspondence between the levels of the intensity scintillation and of the medium drift velocity. The peak values are reached during the equinoxes, in agreement with the scintillation seasonal dependency analysis presented in the previous section. Enhanced values are obtained in particular on occurrence of a magnetic storm. This was the case for the St. Patrick's Day storm of March 2015 (days 80 to 85), which is studied in more detail in Sect. 4 of this paper.

\subsection{High-latitude scintillations}

\subsubsection{Latitudinal and seasonal dependencies}

The results presented use the data recorded with the three $50 \mathrm{~Hz}$ receivers located in Kevo (latitude $69^{\circ} 45^{\prime}$ ), Sodankylä (latitude $67^{\circ} 25^{\prime}$ ) and Kiruna (latitude $67^{\circ} 51^{\prime}$ ). Figure 7 is a cumulative map of the phase scintillation index for the period from January to November 2016. The phase scintillation index is the one calculated by the manufacturer software, i.e. the RMS value after filtering the signal with a sixth-order Butterworth polynomial. The points on the map are the IPP points. The values of the points in each one of the pixels correspond to the average of the highest $10 \%$ of values of the phase scintillation index (the phase RMS value) of the points attached to this pixel. The values, in radians, have been limited to the value of 2 radians. The number of points exhibiting a higher phase scintillation index value is negligible. The cor- 


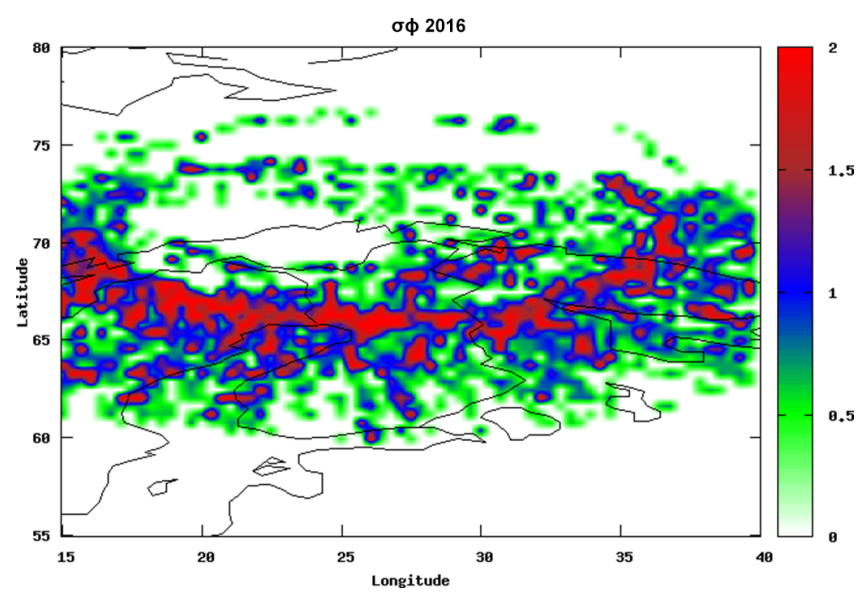

Figure 7. High-latitude phase scintillation cumulative map (phase index in radians).

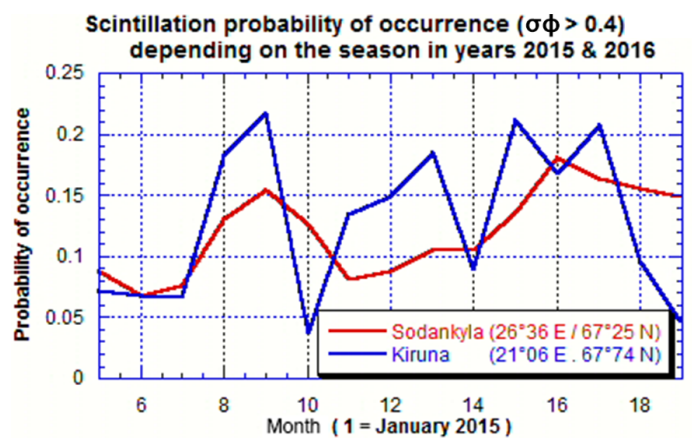

Figure 8. Scintillation seasonal dependency at high latitudes.

responding intensity scintillation map, not reproduced here, shows weak scintillations ( $\mathrm{S} 4<0.2$ in most cases).

The use of the multipath rejection criterion based on the code carrier divergence, as was done for the low-latitude scintillation, leads to the rejection of all links. It has consequently not been implemented for the high-latitude scintillation analysis. A mask elevation angle equal to $20^{\circ}$ was set to reject the multipath with the environment. The analysis shows that there is a clear region of maximum probability of occurrence corresponding approximately to the auroral oval (about $65^{\circ}$ of latitude) and the occurrence of phase scintillation appears limited to the latitudes above $60^{\circ}$. This will be confirmed in the future since we plan to deploy one additional receiver close to Göteborg (southern Sweden). Figure 8 shows the seasonal dependency using the data recorded in 2015 and 2016. The high-latitude scintillation maps do not exhibit an obvious seasonal effect.

\subsubsection{Geophysical dependencies}

The correlation of high-latitude scintillation with the magnetic field has been checked. Figure 9 shows the correspondence between the phase scintillation index and the auroral

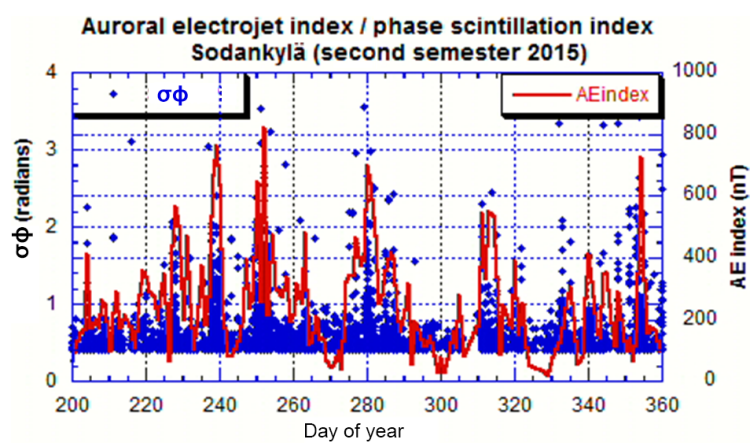

Figure 9. The coincidence of the auroral electrojet index with the phase scintillation index at high latitudes

electrojet (AE) index. High values of the phase scintillation index also correspond to high values of the AE index. A similar analysis was carried out to study the EGNOS days of decrease performance and the correspondence applies to all days, showing the sensitivity of the phase scintillations of the system.

\section{Study case: the St. Patrick's Day storm}

\subsection{Magnetic storm characteristics}

As another example of the MONITOR network and processing capabilities, we present the corresponding analysis performed on the St. Patrick's Day storm that occurred on 17 March 2015. It was the strongest geomagnetic storm of the 24th solar cycle. This storm induced ionospheric effects at both high and low latitudes. It started on 17 March and the effects were noticeable during the whole week following that day. As a reference, the AE index reached a value of $2500 \mathrm{nT}$ and the magnetic activity (Kp) index a value of 8 . This case has been analysed in detail because it is considered representative of extreme events in studies of the ionosphere. The magnetic field components and the AE index are shown in Fig. 10.

The space weather conditions during the event have been detailed in several recent papers (Kamide and Kusano, 2015; J. Liu et al., 2015; Zhang et al., 2015; Cherniak et al., 2015; Jacobsen and Andalsvik, 2016). Figure 10 shows variations in selected interplanetary magnetic field (IMF) and geomagnetic parameters between 15 and 20 March 2015. The sudden storm commencement (SSC) was registered at $\sim 04: 45 \mathrm{UT}$, and there was subsequently a rapid drop in the SYM-H index to $-226 \mathrm{nT}$ at $\sim 23: 00 \mathrm{UT}$, with a couple of local minima of -93 and $-164 \mathrm{nT}$ at $\sim 09: 40$ and $\sim 17: 40$ UT respectively.

The behaviour of the IMF $\mathrm{Bz}$ component is shown in Fig. 10 (top panel). After the SSC, the northward IMF Bz component reached a value of $\sim 25 \mathrm{nT}$. At $\sim 05: 30 \mathrm{UT}$ the IMF $\mathrm{Bz}$ turned southward and reached the first minimum value of $-18 \mathrm{nT}$ at $06: 15 \mathrm{UT}$. Then the IMF $\mathrm{Bz}$ turned 


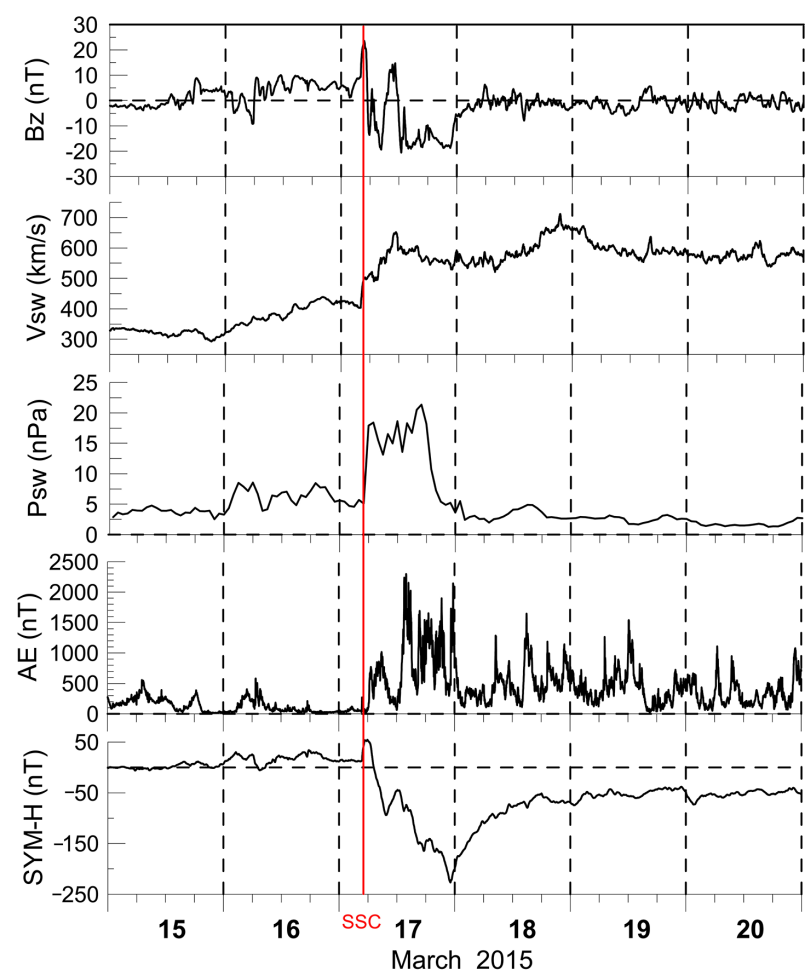

Figure 10. Variations in the interplanetary magnetic field (IMF) and geomagnetic parameters between 15 and 20 March 2015. From top to bottom: IMF Bz component, solar wind speed $\left(V_{\mathrm{SW}}\right)$, solar wind ram pressure $\left(P_{\mathrm{SW}}\right)$, geomagnetic auroral electrojet index $(\mathrm{AE})$ and index of geomagnetic activity (SYM-H). The red line indicates the sudden storm commencement (SSC) time (04:45 UT).

sharply northward and switched extensively between north and south over $\sim 8 \mathrm{~h}$. After $\sim 13: 40$ UT the Bz turned southward again and remained southward until the end of the day.

The AE depicts two intensification peaks at $\sim 09: 00$ and $\sim$ 14:00 UT. Between these two peaks, Bz was observed to turn northward. The mid-latitude magnetic $\mathrm{Kp}$ index (not shown here) reached a value of 8 . The strong disturbance of the geomagnetic field on 17 March 2015 led to an intense particle precipitation and to an enhancement in the substorm activity. It was reported that between 17 and 18 March 2015 auroras were observed at different locations around the globe, even at mid-latitudes as equatorward as Tasmania and New Zealand in the Southern Hemisphere, as well as in the United States, Europe and Japan in the Northern Hemisphere (e.g. T. C. Liu et al., 2015; Kamide and Kusano, 2015; Nishitani et al., 2015; Shiokawa and Otsuka, 2015; GUVI TIMED JHU/APL, 2015; USGS NGP website http://geomag.usgs. gov/storm/22, 2015, and links therein).

The ionospheric characterization capabilities of the MONITOR data and processing network will be exemplified in the St. Patrick's Day geomagnetic storm in the next subsections devoted namely to a. evolution of the rate of total electron content index (ROTI) maps, which provide a very useful proxy of the ionospheric scintillation activity from dual-frequency GNSS carrier phase measurements, complementing the direct high-rate scintillation measurements provided by the MONITOR GNSS receiver network at low and high latitudes (see previous Sects. 3 and 4). In this regard, two kinds of maps are continuously generated in the MONITOR project: a north polar map, a very relevant region for space weather monitoring, and global ROTI maps provided each $15 \mathrm{~min}$ interval in real-time from the corresponding IGS network.

b. evolution of the global VTEC and its overall spatial integral, the global electron content (GEC), obtained from the global ionospheric maps (GIMs), computed by the MONITOR consortium (UPC) since 1998 as an open product on behalf of the IGS. In the MONITOR context, the UPC GIMs, under the identification "UQRG" and computed by tomography and kriging modelling (Hernández-Pajares et al., 1999; OrúsPérez et al., 2005), have been improved, presently being the most or some of the most accurate GIMs (see Hernández-Pajares et al., 2016; Orús-Pérez, 2016). Such global VTEC maps give a unique view of the dynamics of the electron content at a global scale, with VTEC maps provided each $15 \mathrm{~min}$, with a latency of 1 day.

c. assimilation of UQRG GIMs in the NeQuick model in order to try to reproduce the observed TEC rates as closely as possible (in particular with the AATR).

d. the direct observation of scintillation indices at a high latitude, i.e. a privileged place for directly observing space weather effects (Sodankylä), is provided.

e. the real-time detection and measurement of solar flares, frequent precursors of major geomagnetic storms, thanks to the new techniques SISTED and GSFLAI (Hernández-Pajares et al., 2012), developed coinciding with the beginning of the MONITOR project. They convert the global network of GNSS receivers in a solar instrument for detection and for measuring the extreme ultraviolet (EUV) rate.

f. MONITOR also provided the abovementioned AATR for EGNOS RIMS stations as a heuristic proxy of the potential error modelling.

\subsection{ROTI maps}

Figure 11 presents the polar ROTI maps obtained for a regular day (16 March), then for the day of the storm occurrence (17 March) and for the following days. The calculation 


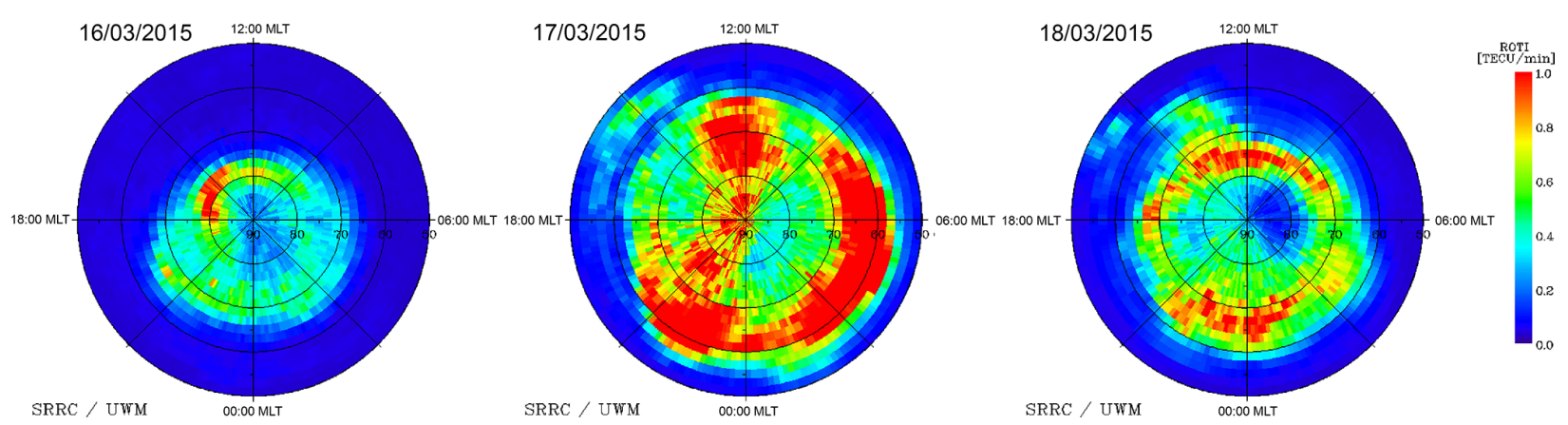

Figure 11. The polar ROTI maps for the St. Patrick's Day storm period (16 to $18 \mathrm{March}$ ).
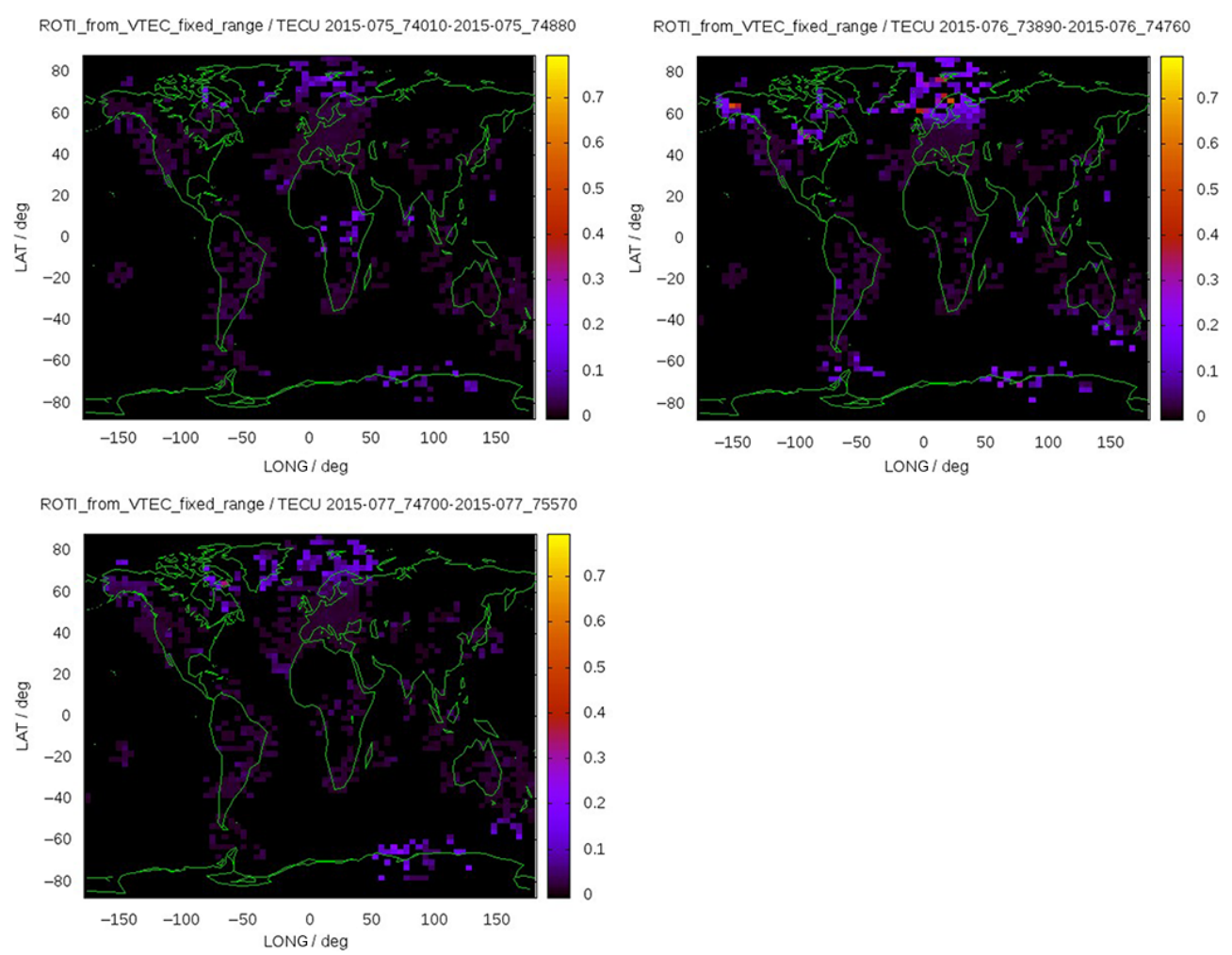

Figure 12. The global ROTI maps for the St. Patrick's Day storm period (16, 17 and 18 March, around 20:45 UT).

is based on the analysis of carrier phase delays in the dualfrequency GPS signals when they passed through the ionospheric plasma. Pi et al. (1997) introduced two indices for ground-based GPS observations: ROT and ROTI. ROT (rate of TEC change) is the time derivative of TEC and is considered to be a measure of phase fluctuation activity. ROT is determined by taking the ratio of the difference between STEC values on two successive occasions according to the time interval. ROT is calculated for each visible GPS satellite over a ground-based GPS station in units of TECU per minute where $1 \mathrm{TECU}=10^{16}$ electrons $\mathrm{m}^{-2}$. ROTI, a standard deviation of ROT, measures the irregular structure of TEC spatial gradients and is used to characterize the severity of GPS phase fluctuations and detect the presence of ionospheric ir- regularities. Here, a single-layer model was used based on the assumption that all concentrations of electrons are located in an infinitely thin spherical shell at a height of $350 \mathrm{~km}$; locations of the fluctuations are related to IPPs. All raw GPS data were resampled to $30 \mathrm{~s}$ resolution, with a cutoff elevation angle of $30^{\circ}$. A detailed description of the ROT-ROTI calculation technique using ground-based GPS is presented in Cherniak et al. (2014).

To observe the spatial distribution of the ionospheric irregularities over northern hemispheric mid- and high latitudes, ROTI data were processed from a representative set of 700 permanent GPS stations. The result is visualized in the form of a ROTI map. The ROTI behaviour is represented as a function of a magnetic local time (MLT) and corrected 

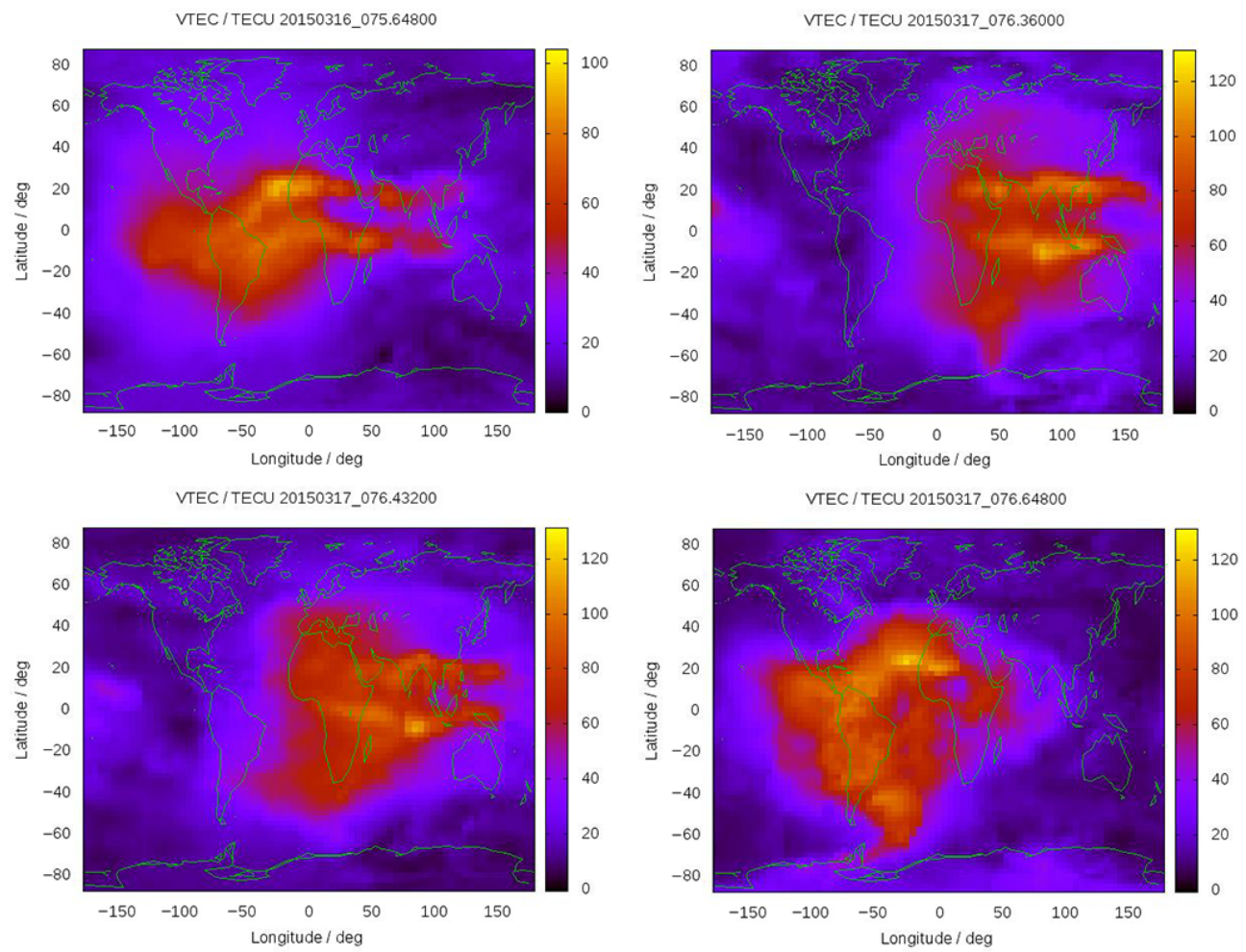

Figure 13. Rapid 15 min resolution VTEC GIMs (labelled UQRG) computed by UPC's TOMION for (from left to right, from top to bottom) 16 March at 18:00 UT and 17 March at 10:00, 12:00 and 18:00 UT.
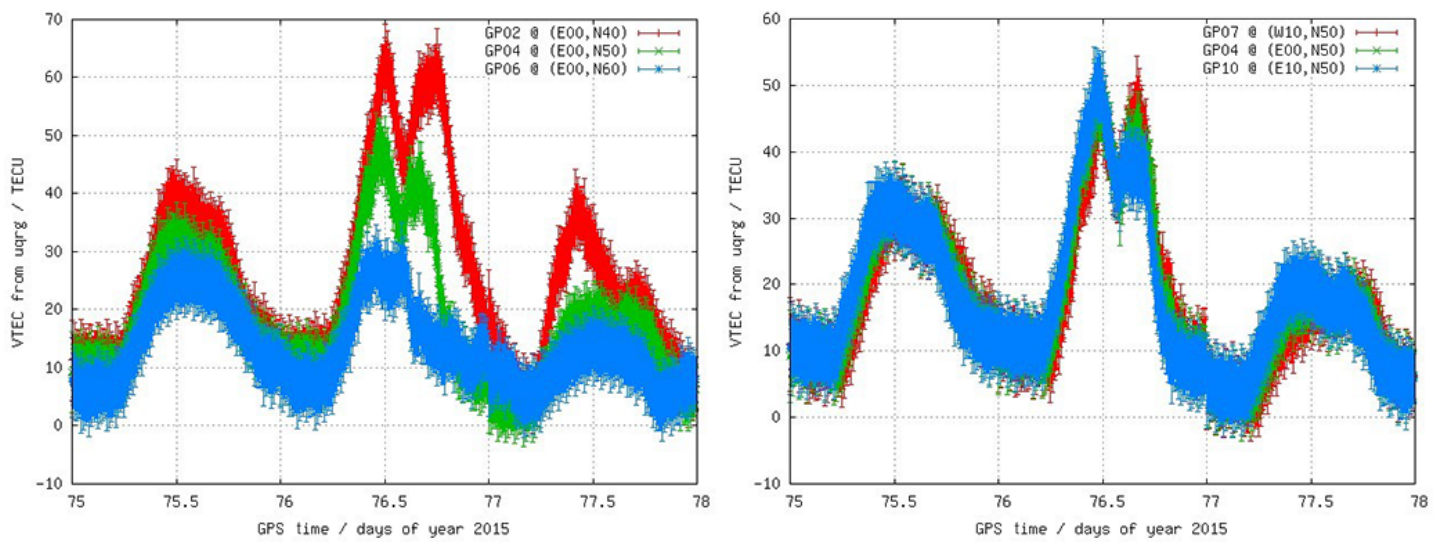

Figure 14. VTEC time series derived from UQRG $15 \mathrm{~min}$ GIMs for the days surrounding the St. Patrick's Day storm (including the previous and next days) for the grid points at $0^{\circ}$ longitude (left-hand plot), with latitudes of 40,50 and $60^{\circ}$ (red, green and blue points) and for the grid points at $50^{\circ}$ latitude (with longitudes of $-10,0$ and $+10^{\circ}$ ).

magnetic latitude (MLAT) for a specific day. Corrected geomagnetic (CGM) coordinates were used with the definite and international geomagnetic reference field (DGRF-IGRF) models. The resultant polar map is a daily map with a 00:0024:00 MLT time frame and the MLAT range of $40-90^{\circ}$. ROTI data were binned and averaged in cells of $2^{\circ}$ MLAT by $8 \mathrm{~min}$ MLT, i.e. the map resolution is 25 cells along the MLAT axis and 180 cells along the MLT axis. The value in every cell is calculated by averaging all ROTI values covered by the cell area and is proportional to the irregularity occurrence probability in the current sector.

For the relatively quiet day of $16 \mathrm{March}$, the position of the irregularities oval is within $75^{\circ}$ MLAT and the polar ROTI values reach their peak intensity around 14:00-16:00 MLT. Intensification of the irregularity occurrence was also observed within $65-70^{\circ}$ MLAT in the pre-midnight sector. The 

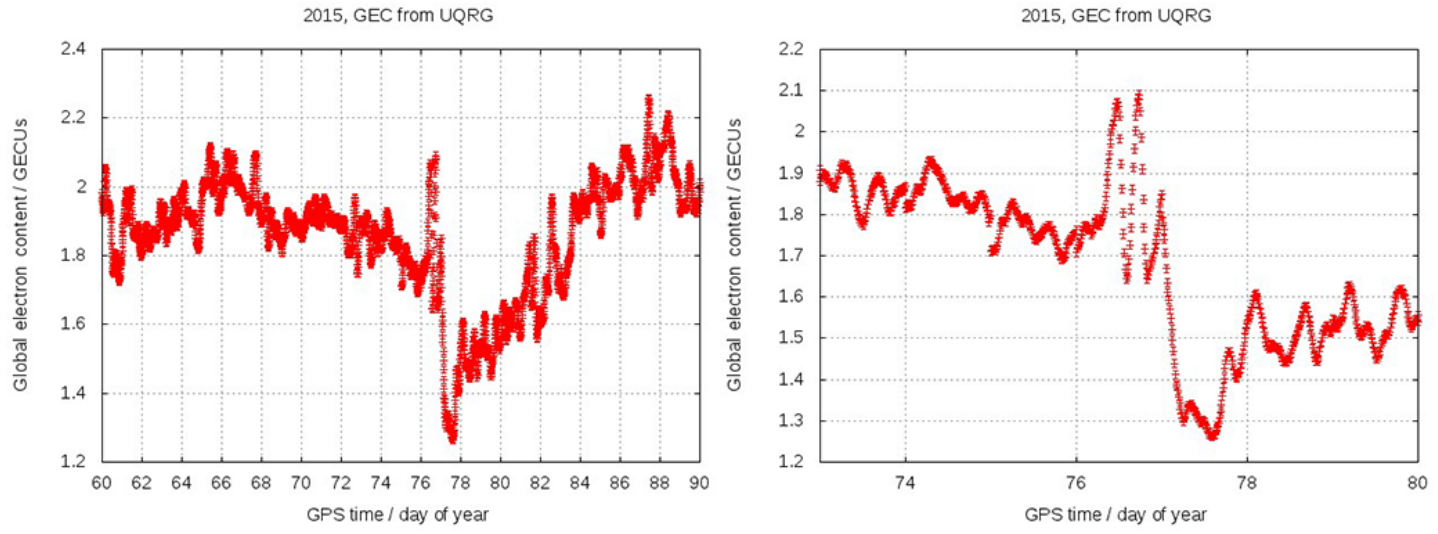

Figure 15. GEC trend derived from UQRG 15 min GIMs for the days surrounding the St. Patrick's Day storm (days 60 to 90 on the left and zoom on days 73 to 80 on the right).

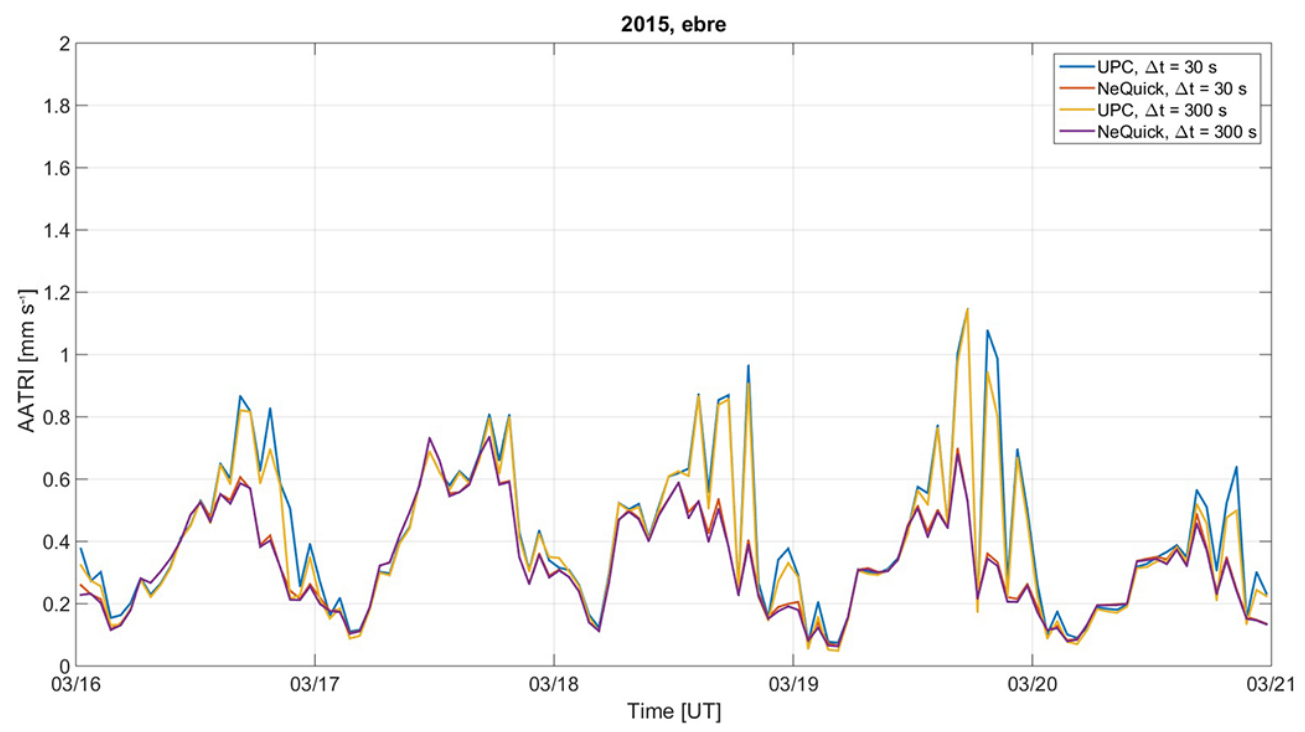

Figure 16. AATR index computed using $\mathrm{d} t=30$ and $300 \mathrm{~s}$ for experimental STEC data and STEC data reconstructed after VTEC data ingestion. St. Patrick's Day storm, March 2015 at Ebre station (Lat $=40.8$, Long $=0.5$ ).

diurnal ROTI map corresponding to the most disturbed day of 17 March 2015 shows significant changes in the pattern of the ionospheric plasma irregularities. A strong increase in the ROTI intensity was accompanied by an expansion of the equatorial border of the irregularities oval (practically to $55^{\circ}$ MLAT). In the polar ROTI maps for this day it is possible to recognize the radial-oriented structures in the day-night direction. These structures can be associated with the formation of the SED-TOI (storm-enhanced density and tongue of ionization) structures and their further transformation into large-scale polar cap patches, which follow the convection pattern anti-sunward across the polar cap (Cherniak et al., 2015).

The corresponding results with the global maps of ROTI (another real-time product of the MONITOR project also computed from the GNSS data streams provided by IGS; see
Caissy et al., 2011) can be seen in Fig. 12 for three similar epochs at the end of the days 16, 17 and 18 March. Much higher ROTI values are found in northern Europe and North America at the end of day 17, in agreement with the occurrence of the geomagnetic storm and previous polar ROTI maps.

\subsection{TEC maps and GEC evolution}

The development of the storm was characterized by a positive phase peak in electron density followed by a negative peak. The strong decrease in electron content over Europe, coinciding with the virtual disappearance of the equatorial anomaly can be seen on the selected VTEC snapshots shown in Fig. 13 (in particular, the VTEC distribution at 18:00 UT can be compared between the day of the storm and the previous day). 
S4 and $\sigma \phi$ on L1 (High latitudes) St. Patrick's Day storm: days 75-82 2015

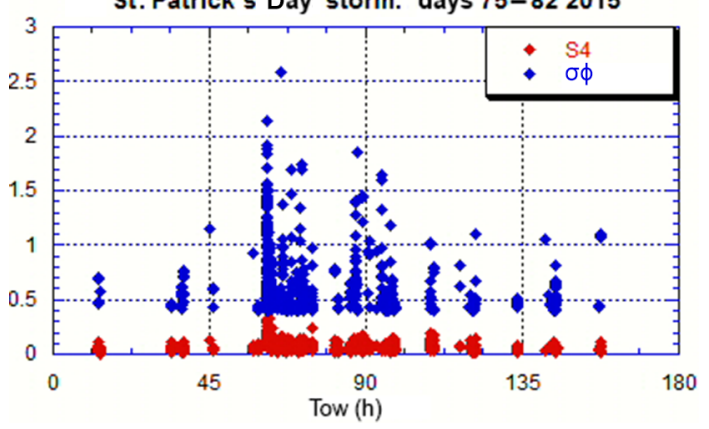

Figure 17. High-latitude scintillation recorded in Sodankylä (Finland).

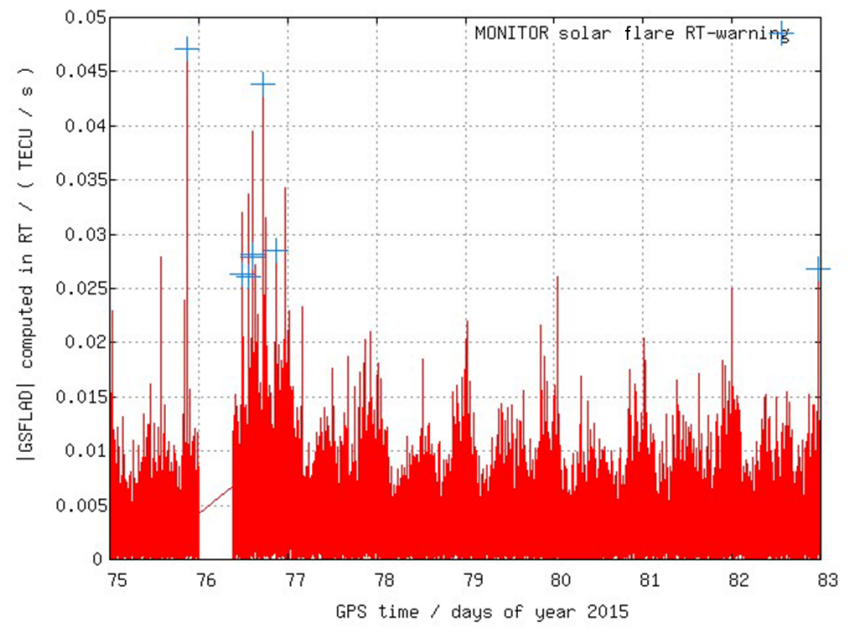

Figure 18. Absolute value of GSFLAI index as it was computed in real time on days $75-82,2015$. The blue crosses indicate the solar flare warnings provided by the UPC system in real time).

This global map UQRG is the rapid UPC VTEC map produced at $15 \mathrm{~min}$ temporal resolution introduced above.

These variations of electron content can be seen clearly in Fig. 14, containing the VTEC time series extracted from the same GIM for three grid points on the same meridian over Europe (left plot) and for three points on the same parallel over Europe (Fig. 14, right plot). The most extreme variations can be seen among the lowest latitudes and during the negative phase (day 77, after the onset of the storm) at the highest latitudes. Regarding the longitude, the results are much more similar, except for the second daily peak during the storm day (76), which is most significant in the west.

The positive phase peak in electron density at a global scale can be better characterized by observing the GEC (Afraimovich et al., 2008) trend in Fig. 15. The detailed view shows two important GEC increases $(+20 \%)$, separated by $6 \mathrm{~h}$, followed by a deep negative phase (almost $-25 \%$ ) $\sim 18$ h later.

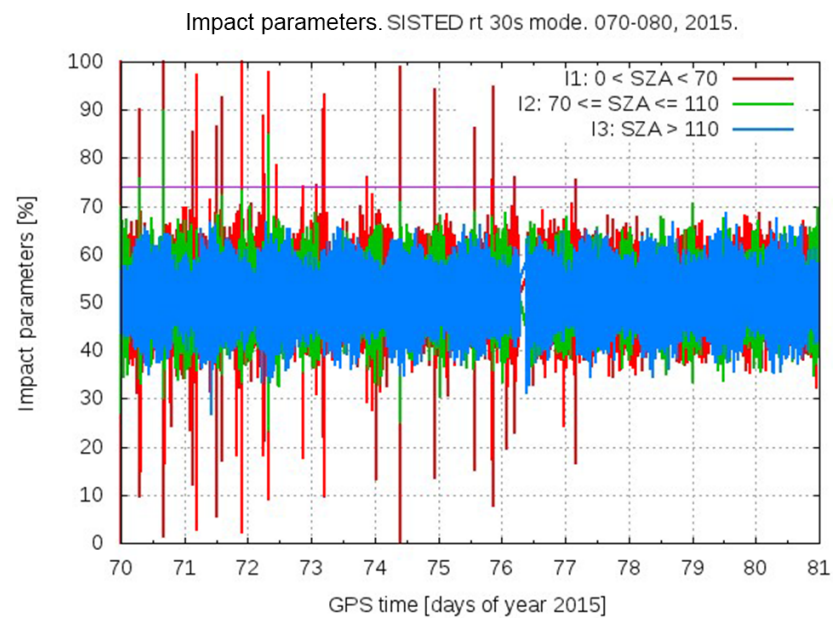

Figure 19. SISTED impact parameters evolution on days 70-80, 2015. The sunlit ionospheric region values are marked in red, the dawn and dusk values in green and the nighttime ones in blue.

\subsection{Data assimilation in the NeQuick model}

To evaluate the data ingestion technique described in Sect. 2 during a geomagnetically disturbed period, the ionospheric scenario generated using UPC VTEC maps for the period of the St. Patrick's Day storm in 2015 has been considered. Specific tests using AATR (Sanz et al., 2014) and ROT indices were performed. In particular, the AATR index was computed for the available receivers using experimental TEC data and was then compared to the same AATR index as computed with the corresponding reconstructed TEC data (after VTEC data ingestion). In Fig. 16, AATR index computed with 30 and $300 \mathrm{~s}$ time interval using experimental (blue and yellow lines respectively) and reconstructed (orange and purple lines respectively) data from the Ebre GNSS station are presented. It can be seen from the plot that AATR index obtained from reconstructed data qualitatively represents the AATR index computed using experimental data well, not only during quiet days (16-17 March) but also on the geomagnetically disturbed days (18-20 March). Nevertheless, the reconstructed index values might differ from the experimental ones, especially during the periods of high TEC fluctuations. This fact can be explained by the use of data for the ingestion with a sampling interval greater than the representative TEC fluctuation periods. The tests performed indicated that the generated scenario is able to represent the ionospheric behaviour in a realistic way and that the reconstructed ionosphere does not introduce remarkable artificial ROT and/or AATR. As expected, the scenario is not able to perfectly reproduce the real ionospheric behaviour, especially if rapid STEC variations are considered (indeed the source of data ingested into the model is VTEC maps at 15 min time intervals). 

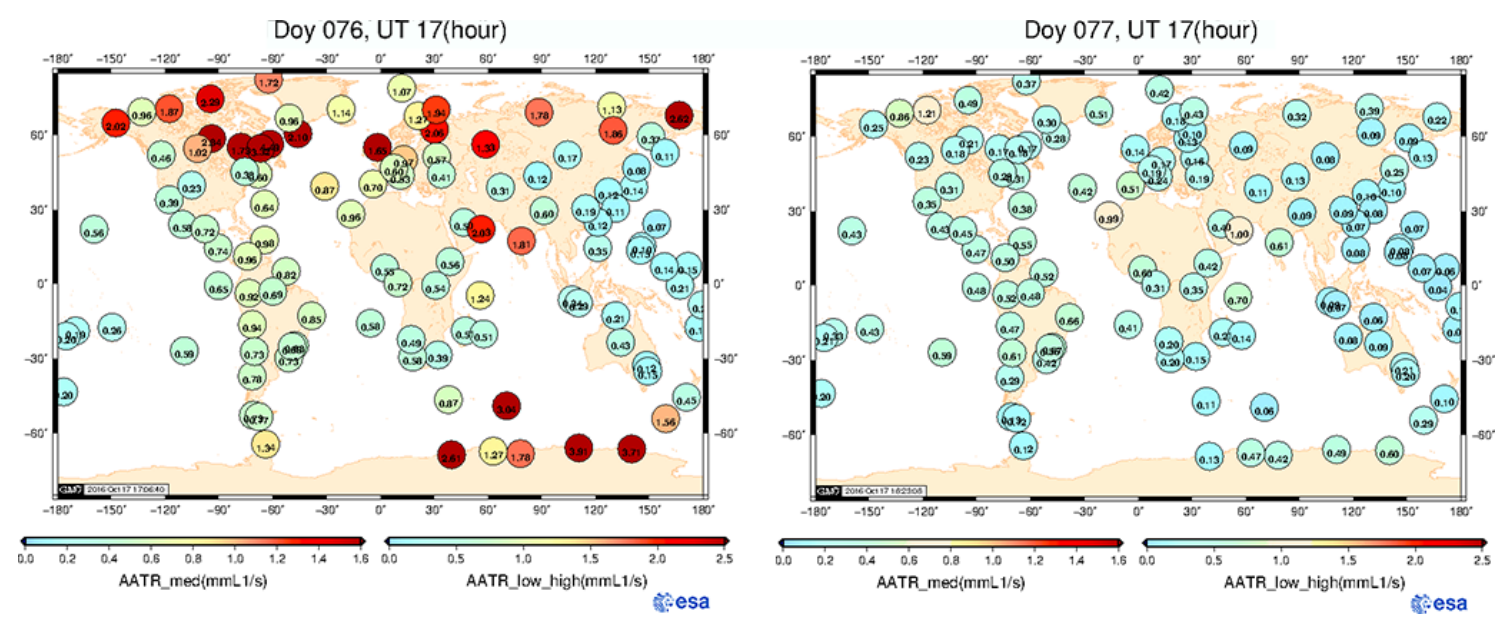

Figure 20. Global AATR for 17 (left) and 18 (right) March 2015 at 17:00 extracted from MONITOR products (http://monitor.estec.esa.int).
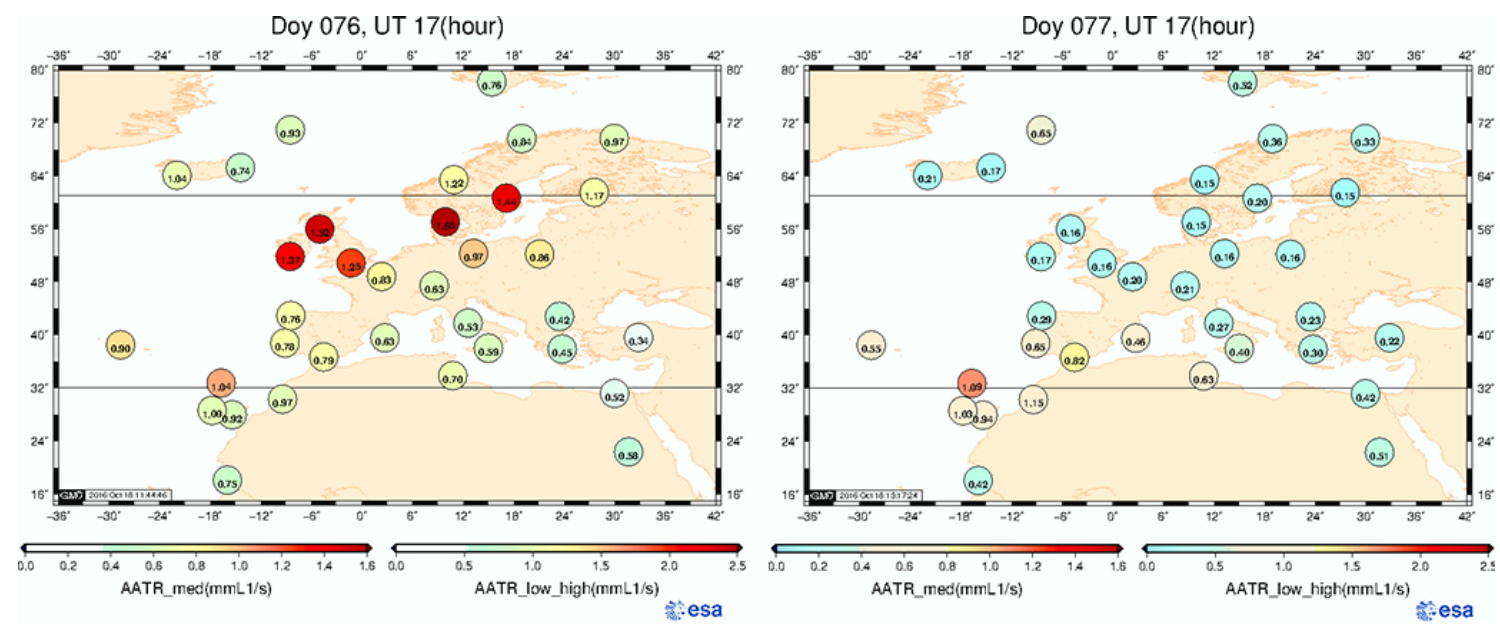

Figure 21. EGNOS RIMS AATR for 17 (left) and 18 (right) March 2015 at 17:00 extracted from MONITOR products (http://monitor.estec. esa.int).

\subsection{Scintillations}

The high-latitude scintillation index recorded in Sodankylä is plotted in Fig. 17. The time origin is the 15 March at 00:00 UT. This timescale is identical to the timescale used in Fig. 10. Strong scintillation activity is clearly correlated to large values of the AE index (see Fig. 10).

As usually observed at high latitudes, the intensity index correspondingly has a rather low value.

\subsection{Solar flares}

Several geoeffective solar flares occurred from days 70 to 76, 2015 (11 to 17 March), prior to the St. Patrick's Day storm, and these were detected and notified in real time by the MONITOR system by means of the GNSS solar flare indicator, (GSFLAI; Hernández-Pajares et al., 2012; MonteMoreno and Hernández-Pajares, 2014; Singh et al., 2015) and the sunlit ionosphere sudden TEC enhancement detector (SISTED; García-Rigo, 2012). Figures 18 and 19 show the corresponding plots for the days surrounding the St. Patrick's Day storm. Indeed, Fig. 18 shows the real-time records of the absolute value of GSFLAI, i.e. the rate of sudden overionization increase (obtained from worldwide dualfrequency GPS measurements) vs. (the cosinus of) the solarionospheric pierce point angle. Fig. 19 shows the SISTED index for a period containing the St. Patrick's Day storm (SISTED mainly shows the predominant increase in ionization occurring at solar-ionospheric pierce point angles less than $70^{\circ}$ shown by the red peaks above the percentage threshold). The records obtained were in agreement with direct GOES (Geostationary Operational Environmental Satellite) observations in the X-ray band. 


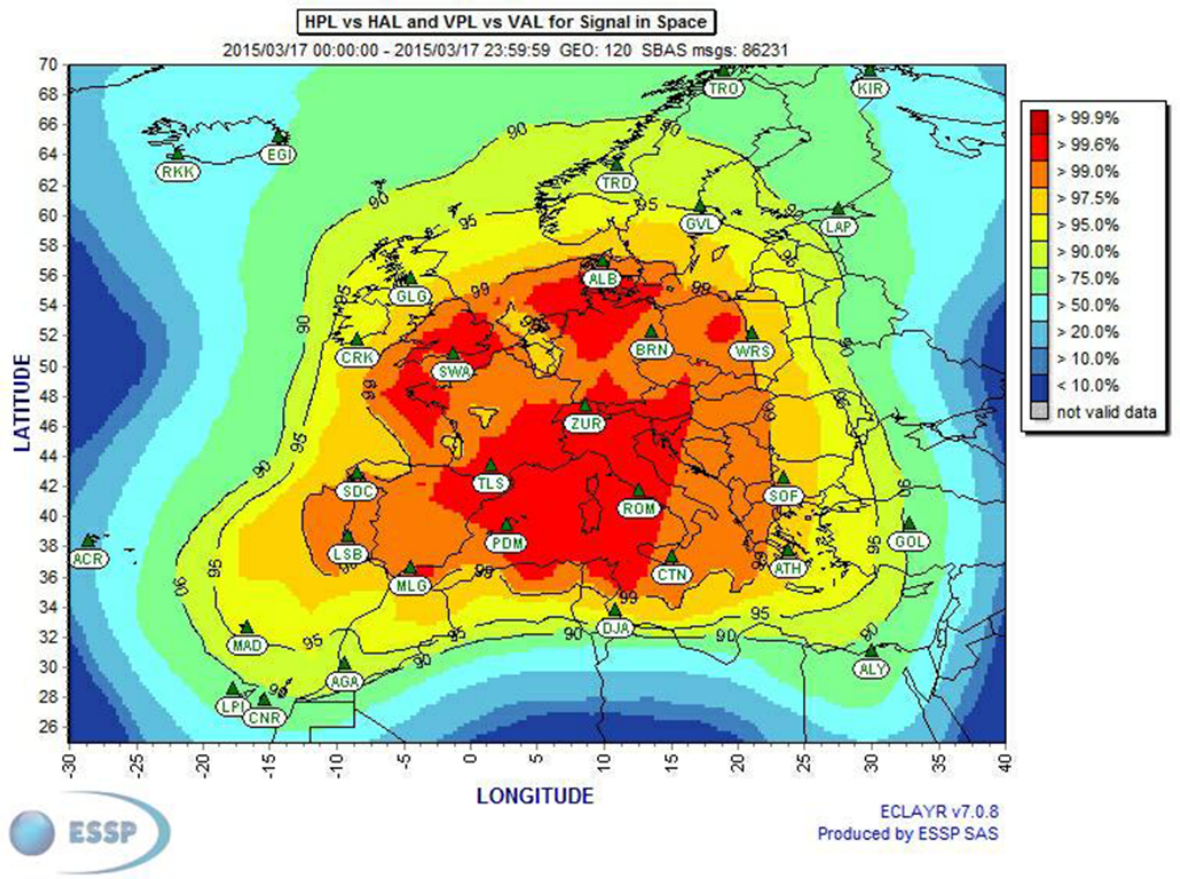

Figure 22. APV-I availability maps for the period of the St. Patrick's Day storm for the 17 and 18 March 2015 (credits: ESSP; https: //egnos-user-support.essp-sas.eu/new_egnos_ops/apv1_availability).

\subsection{RMS AATR maps and EGNOS performances}

Figure 20 shows the global RMS AATR at 1 critical hour for a global network of IGS receivers. Two extracts at 17:00 UT are shown for the St. Patrick's Day storm day and the following day. It is clear that the storm had a very significant effect on the IGS receivers, mostly at medium and high latitudes. The thresholds of the data were defined by Sanz et al. (2014), who have performed a study during a whole solar cycle to specify the $99 \%$ values. These values are 1.6 and $2.5 \mathrm{~mm} \mathrm{~s}^{-1}$ for medium and high or low latitudes respectively.

The MONITOR project also routinely computes the RMS AATR for the EGNOS RIMS. These plots are useful to see whether low APV-I availabilities may be caused by ionospheric perturbations or not. This EGNOS APV-I availability is defined as the percentage of epochs in which the protection level is below alert limits for this APV-I service (horizontal protection level (HPL) $<40 \mathrm{~m}$ and vertical protection level $(\mathrm{VPL})<50 \mathrm{~m}$ ) over that period. Therefore, the St. Patrick's Day storm was a good opportunity to check the results with the RMS AATR maps provided by MONITOR and compare them with the APV-I availabilities of EGNOS. In Fig. 21, the RMS AATR for 17 and 18 March 2015 shows strong activity on the high-latitude receivers and, to a minor extent, on the low-latitude receivers. Figure 22 shows that the zones with high RMS AATR also have a reduced APV-I availability. This implies that at roughly $1 \mathrm{~mm} \mathrm{~s}^{-1}$ of RMS AATR, one could probably expect a decrease in EGNOS APV-I availability.

\section{Conclusions}

The MONITOR + SAGAIE network is the largest ionospheric study network in the western African low-latitude region. It covers longitudes from -20 to $+30^{\circ}$. The global MONITOR ionospheric data and processing network working since 2010 has been summarized and illustrated in this paper. The scintillation measurement campaign reported has lasted almost 2 years for most of the receivers deployed, making it possible to obtain the ionospheric seasonal variability. This project in its last period was conducted to support the EGNOS project. New products focusing on the needs of EGNOS have been developed. The magnetic storms were found to be the most critical events for EGNOS and we checked the capability of the processors developed against the St Patrick's Day event of March 2015, which was the major magnetic storm of the 24th solar cycle. The analysis presented in this document has shown that all processors detected this event and have therefore provided a clear understanding of the ionospheric variability pertaining to this particular case.

Data availability. The URLs of the data used have been indicated in the text when publicly accessible.

Competing interests. The authors declare that they have no conflict of interest. 
Acknowledgements. The MONITOR project was launched by ESA/ESTEC in 2010 (contract no. 4000100988). The second phase of the study to support the EGNOS project was launched in 2014 by ESA/EGNOS Project Office. The authors want to acknowledge IGS for providing ionospheric data measurements and products (in real time), BKG/CNES for NTRIP real-time GNSS data streams and the NASA/GSFC's Space Physics Data Facility's OmniWeb service for providing OMNI data (ftp://spdf.gsfc.nasa.gov/pub/data/ omni) and the program code for calculating the CGM coordinates. The AE index was provided by the World Data Center for Geomagnetism at Kyoto University (http://wdc.kugi.kyoto-u.ac.jp). The authors would like to thank B. Arbesser and R. Prieto-Cerdeira (ESA/ESTEC), who launched this project, S. Scortan and A. Grosu from CS-Romania, ASECNA, CNES SAGAIE, the Finnish Meteorological Institute (FMI), QinetiQ, the University of Birmingham and all those who have contributed to making the MONITOR project a success.

The authors would also like to thank the reviewers for many helpful remarks and comments.

The topical editor, J. Makela, thanks K. S. Jacobsen and the one anonymous referee for help in evaluating this paper.

\section{References}

Afraimovich, E. L., Astafyeva, E. I., Oinats, A. V., Yasukevich, Yu. V., and Zhivetiev, I. V.: Global electron content: a new conception to track solar activity, Ann. Geophys., 26, 335-344, doi:10.5194/angeo-26-335-2008, 2008.

Béniguel, Y., Hernandez-Pajares, M., Garcia-Rigo, A., Orus-Perez, R., Prieto-Cerdeira, R., Schlueter, S., Secretan, H., Monnerat, M., and Serant, D.: MONITOR Ionospheric Monitoring System: GNSS Performance Estimation, European Navigation Conference, Bordeaux, April 2015.

Caissy, M., Weber, G., Agrotis, L., Wübbena, G., and HernandezPajares, M.: The IGS real-time pilot project - the development of realtime IGS correction products for precise point positioning, Geophys. Res. Abstr., vol. 13, EGU2011-7472, EGU General Assembly 2011, Vienna, Austria, 2011.

Carrano, C., Groves, K., Rino, C., and Doherty, P.: A technique for inferring Zonal Irregularity Drift from Single-Station GNSS Measurements of intensity (S4) and Phase $\left(\sigma_{\Phi}\right)$ Scintillations, Radio Sci., 51, 1263-1277, doi:10.1002/2015RS005864, 2016.

Cherniak, I., Krankowski, A., and Zakharenkova, I.: Observation of the ionospheric irregularities over the Northern Hemisphere: Methodology and service, Radio Sci., 49, 653-662, doi:10.1002/2014RS005433, 2014.

Cherniak, I., Zakharenkova, I., and Redmon, R.: Dynamics of the high-latitude ionospheric irregularities during the March 17, 2015 St. Patrick's Day storm: Ground-based GPS measurements, Space Weather, 13, 585-597, doi:10.1002/2015SW001237, 2015.

Feltens, J., Angling, M., Jackson Booth, N., Jakowski, N., Hoque, M., Hernández Pajares, M., Aragón Àngel, A., Orús, R., and Zandbergen, R.: Comparative testing of four ionospheric models driven with GPS measurements, Radio Sci., 46, RS0D12, doi:10.1029/2010RS004584, 2011.

García-Rigo, A.: Contributions to ionospheric determination with global positioning system: solar flare detection and prediction of global maps of total electron content, $\mathrm{PhD}$ dissertation, Universitat Politècnica de Catalunya, Barcelona, Spain, 2012.

GUVI TIMED JHU/APL: guvitimed.jhuapl.edu, available at: http: //guvitimed.jhuapl.edu/guviimages/ (last access: 28 September 2016), 2015.

Hernández-Pajares, M., Juan, J. M., and Sanz, J.: New approaches in global ionospheric determination using ground GPS data, J. Atmos. Sol.-Terr. Phys., 61, 1237-1247, 1999.

Hernández-Pajares, M., García-Rigo, A., Juan, J. M., Sanz, J., Monte, E., and Aragón-Àngel, A.: GNSS measurement of EUV photons flux rate during strong and mid solar flares, Space Weather, 10, S12001, doi:10.1029/2012SW000826, 2012a.

Hernández-Pajares, M., Juan, J. M., Sanz, J., and Aragón-Àngel, A.: Propagation of medium scale traveling ionospheric disturbances at different latitudes and solar cycle conditions, Radio Sci., 47, RS0K05, doi:10.1029/2011RS004951, 2012b.

Hernández-Pajares, M., Prieto-Cerdeira, R., Béniguel, Y., GarciaRigo, A., Kinrade, J., Kauristie, K., Orus-Perez, R., Schlüter, S., Serant, D., Nava, B., Krankowski, A., Secretan, H., Sampedro, R., and Prats, X.: MONITOR ionospheric monitoring system: analysis of perturbed days affecting SBAS performance, ION PNT, Honolulu, 2015.

Hernández-Pajares, M., Roma-Dollase, D., Krankowski, A., Ghoddousi-Fard, R., Yuan, Y., Li, Z., Zhang, H., Shi, C., Feltens, J., Komjathy, A., Vergados, P., Schaer, S., Garcia-Rigo, A., and Gómez-Cama, J. M.: Comparing performances of seven different global VTEC ionospheric models in the IGS Context, in: IGS Workshop, 8-12 February 2016, Sydney, Australia, International GNSS Service (IGS), 1-31, 2016.

Jacobsen, K. S. and Andalsvik, Y. L.: Overview of the 2015 St. Patrick's day storm and its consequences for RTK and PPP positioning in Norway, Journal of Space Weather and Space Climate, 6, A9, doi:10.1051/swsc/2016004, 2016.

Kamide, Y. and Kusano, K.: No Major Solar Flares but the Largest Geomagnetic Storm in the Present Solar Cycle, Space Weather, 13, 365-367, doi:10.1002/2015SW001213, 2015.

Liu, J., Wang, W., Burns, A., Yue, X., Zhang, S., Zhang, Y., and Huang, C.: Profiles of ionospheric storm-enhanced density during the 17 March 2015 great storm, J. Geophys. Res.-Space, 121, 727-744, doi:10.1002/2015JA021832, 2015.

Liu, T. C., Shao, X., Cao, C., Zhang, B., Fung, S. F., and Sharma, S.: Aurora Activities Observed by SNPP VIIRS Day-Night Band during St. Patrick's Day, 2015 G4 Level Geomagnetic Storm, AGU Fall Meeting, San Francisco, USA, 14-18 December 2015, SA31D-2360, 2015.

Monte-Moreno, E. and Hernández-Pajares, M.: Occurrence of solar flares viewed with GPS: Statistics and fractal nature, J. Geophys. Res.-Space, 119, 9216-9227, doi:10.1002/2014JA020206, 2014

Nava, B., Coïsson, P., and Radicella, S. M.: A new version of the NeQuick ionosphere electron density model, J. Atmos. Sol.-Terr. Phys., 70, 1856-1862, doi:10.1016/j.jastp.2008.01.015, 2008.

Nava, B., Radicella, S. M., and Azpilicueta, F.: Data ingestion into NeQuick 2, Radio Sci., 46, RS0D17, doi:10.1029/2010RS004635, 2011.

Nishitani, N., Hori, T., Kataoka, R., Ebihara, Y., and Shiokawa, K.: Characteristics of ionospheric convection associated with lowlatitude aurora observed at Rikubetsu, Hokkaido during the 2015 March storm, SuperDARN workshop 2015, Leicester, UK, 2015. 
Orús-Pérez, R.: Ionospheric error contribution to GNSS singlefrequency navigation at the 2014 solar maximum, J. Geodesy, 1-11, doi:10.1007/s00190-016-0971-0, online first, 2016.

Orús-Pérez, R., Hernández-Pajares, M., Juan, J. M., and Sanz, J.: Improvement of global ionospheric VTEC maps by using kriging interpolation technique, J. Atmos. Sol.-Terr. Phy., 67, 15981609, 2005.

Pi, X., Mannucci, A. J., Lindqwister, U. J., and Ho, C. M.: Monitoring of global ionospheric irregularities using the worldwide GPS network, Geophys. Res. Lett., 24, 2283-2286, 1997.

Rino, C.: A power law phase screen model for ionospheric scintillations (weak scatter), Radio Sci., 14, 1135-1145, 1979.

Rino, C.: The Theory of Scintillation with Applications in Remote Sensing, Wiley, IEEE Press, 2011.

Sanz, J., Juan, J. M., González-Casado, G., Prieto-Cerdeira, R., Schlüter, S., and Orús-Perez, R.: Novel Ionospheric Activity Indicator Specifically Tailored for GNSS Users, ION GNSS 2014, Tampa, 2014.

Secrétan, H., Monnerat, M., and Kameni, R.: SAGAIE, a GNSS Network for Investigating Ionospheric behavior in sub-Saharan region, Inside GNSS, 9, 46-58, 2014.
Shiokawa, K. and Otsuka, Y.: Low-latitude red aurora observed in Japan during the St. Patrick's Day 2015 Event, SCOSTEPWDS Workshop on Global Data Activities for the Study of SolarTerrestrial Variability, NICT, Tokyo, Japan, 28-30 September 2015, Book of Abstracts, p. 34, 2015.

Singh, T., Hernandez-Pajares, M., Monte, E., Garcia-Rigo, A., and Olivares-Pulido, G.: GPS as a solar observational instrument: Real-time estimation of EUV photons flux rate during strong, medium, and weak solar flares, J. Geophys. Res.-Space, 120, 10840-10850, doi:10.1002/2015JA021824, 2015.

Xiang, Y., Yuan, Y., Li, Z., and Wang, N.: Analysis and validation of different global ionospheric maps (GIMs) over China, Adv. Space Res., 55, 199-210, 2015.

Zhang, S., Erickson, P. J., Foster, J. C., Holt, J. M., Coster, A. J., Makela, J. J., Noto, J., Meriwether, J. W., Harding, B. J., Riccobono, J., and Kerr, R. B.: Thermospheric poleward wind surge at mid latitudes during great storm intervals, Geophys. Res. Lett., 42, 5132-5140, doi:10.1002/2015GL064836, 2015. 